\title{
Evaluation of the Adaptation and Fracture Resistance of Three CAD-CAM Resin Ceramics: An In vitro Study
}

\author{
Nicolas Naffah ${ }^{1}$, Hani Ounsi ${ }^{2}$, Mutlu Ozcan ${ }^{3}$, Hsein Bassal ${ }^{4}$, Ziad Salameh $^{5}$
}

\begin{abstract}
Aim: The internal fit and resistance to fracture of resin ceramics are to be evaluated compared to that of lithium disilicate as the control group. Materials and methods: Four groups of 20 crowns each (GC Cerasmart, Vita Enamic, Coltène Brilliant Crios, and e.max CAD) were cemented on identical metal dies. Marginal gaps were measured before cementation and load to fracture was applied after cementation, half of each group was thermodynamically aged (3,000 cycles of $5^{\circ}$ to $55^{\circ}$ immersion followed by 200,000 cycles of $100 \mathrm{~N}$ load), finally the crowns were loaded until fracture in a universal testing machine. Statistical package for social sciences (SPSS) package 23 was used for statistical work.

Results: Marginal gaps ranged between $68.5 \pm 23.8 \mu \mathrm{m}$ and $87 \pm 29.1 \mu \mathrm{m}$ while occlusal gaps ranged from $220.7 \pm 33.3 \mu \mathrm{m}$ to $275.5 \pm 46.5 \mu \mathrm{m}$ and were not significantly different between groups. Fracture loads ranged from $633.8 \pm 127.3 \mathrm{~N}$ to $1596.4 \pm 497.7 \mathrm{~N}$ with lithium disilicate glass ceramics (LDGCs) and Enamic having higher values than resin nano-ceramics (RNCs). The fracture resistance was more related to material than aging and gap value.

Conclusion: The margin adaptation of resin ceramics was comparable to lithium disilicate with no significant difference. Lithium disilicate showed a higher resistance than resin ceramics and there was a higher resistance to fracture for polymer-infiltrated ceramic-network (PICN) than RNCs.

Clinical significance: Resin ceramics can have marginal adaptation and fracture resistance within clinical acceptance; therefore, they can be a good chair-side solution achieved in a single appointment session.

Keywords: Fracture resistance, Internal fit, Lithium disilicate, Resin ceramics.

The Journal of Contemporary Dental Practice (2019): 10.5005/jp-journals-10024-2560
\end{abstract}

\section{INTRODUCTION}

Computer-aided design-computer-aided manufacturing (CAD-CAM) materials have offered numerous advantages in the field of dentistry compared with conventional crown fabrication in terms of time, precision, ${ }^{1}$ and laboratory skill dependency. ${ }^{2}$ CAD-CAM glass ceramic blocks, or lithium disilicate glass ceramics (LDGC, IPS e.max, IVOCLAR VIVADENT), were first introduced in an attempt to standardize workflow quality (material and shade) and have demonstrated high survival rates and aesthetic success over time. ${ }^{3}$ However, their milling is both time and bur consuming ${ }^{4}$ and can generate cracks and edge chipping. ${ }^{5}$ The more recently introduced resin-based CAD-CAM blocks/disks have been designed as alternatives to CAD-CAM glass ceramics, they are easier to mill, provide a smoother final surface, and require fewer manufacturing steps.., 5

There are two manufacturing techniques for resin ceramic blocks. Resin nano-ceramics (RNCs) comprise highly charged dispersed nanoceramic particles in a polymer network. Polymer-infiltrated ceramicnetwork (PICN) materials involve infiltrating a pre-existing ceramic network with resin polymers. The diversity in resin ceramic ingredients and network design provides these materials with comparatively high flexural strength ${ }^{6}$ that results in reduced chipping and fractures when compared to glass ceramics. The resilient resinous matrix acts as a shock absorber while the highly dense ceramic charge gives the material its strength and possible hydrofluoric acid surface treatment. ${ }^{7}$

A proper marginal fit is crucial to ensure minimal cement film thickness, poor adaptation being closely associated with gum inflammation, secondary caries, and prostheses failure.' Since the hardness of a CAD/CAM material affects the amount of material removed during milling, ${ }^{6}$ similar materials in structure design may respond differently during the same workflow and result in different gap values. Additionally, different materials have varying
${ }^{1}$ Department of Prosthodontics, Faculty of Dental Medicine, Lebanese University, Beirut, Lebanon

${ }^{2}$ Department of Endodontics, Faculty of Dental Medicine, Lebanese University, Beirut, Lebanon; Faculty of Dental Medicine, Siena University, Siena SI, Italy

${ }^{3}$ Department of Dental Materials, Faculty of Dental Medicine, Zurich University, Zurich, Switzerland

${ }^{4}$ Faculty of Science, Lebanese University, Beirut, Lebanon

${ }^{5}$ Research Center and Department of Prosthodontics, Faculty of Dental Medicine, Lebanese University, Beirut, Lebanon

Corresponding Author: Nicolas Naffah, Department of Prosthodontics, Faculty of Dental Medicine, Lebanese University, Beirut, Lebanon, Phone: +961 3300122, e-mail: drnic961@gmail.com

How to cite this article: Naffah N, Ounsi $\mathrm{H}$, et al. Evaluation of the Adaptation and Fracture Resistance of Three CAD-CAM Resin Ceramics: An In vitro Study. J Contemp Dent Pract 2019;20(5):571-576.

\section{Source of support: Nil}

Conflict of interest: None

structural designs and physical properties and, therefore, do not respond equally to occlusal forces, thermal changes, and fatigue.

The aims of this study are to evaluate (1) the internal fit and fracture resistance of three CAD-CAM resin ceramic materials compared to CAD-CAM lithium disilicate, (2) to evaluate the effect of aging, and (3) to assess the relationship between internal fit and fracture resistance. The null hypotheses tested were that (1) there is no difference in the internal fit and fracture resistance with or without aging between the materials tested and (2) there is no significant relationship between internal fit and fracture resistance. 


\section{Materials and Methods}

\section{Preparation of Die Specimens}

A typodont (Nissin, Japan) upper first premolar was prepared to receive a full-coverage crown. A standardized shoulder of $1 \mathrm{~mm}$ depth with rounded interior angle was formed using a surveyor (IRIS, Tianjin, China). The reduction of the labial, lingual, and occlusal surfaces was at $\geq 1.5 \mathrm{~mm}$ (the occlusal edges were finished without the surveyor), the preparation had a $6^{\circ}$ taper (F850.0108, $25 \mu$ grit EDENTA Dental, Switzerland), and the occlusal angle between the two cusp slopes was $150^{\circ}$ (Fig. 1).

The prepared tooth was removed from the practice model and two wax rings were added to its apical part to form the master die (Fig. 2). The die was then embedded in a duplicating silicon mold (RTV 3040A, Bluesil, Germany) and injected with castable wax (Castaldo, 120 Constitution Boulevard, Franklin, MA 02038, USA) to make 80 replicas that were cast in nickel-chromium $(\mathrm{Ni}-\mathrm{Cr})$ alloy (EX-3 Noritake Super Alloy, Kuraray Noritake Dental Inc, Miyoshi, Aichi, Japan). This resulted in $80 \mathrm{Ni}-\mathrm{Cr}$ dies identical to the original preparation. All metal dies were examined (air bubbles and distortions) using a magnifying stereoscope $(\times 20)$ (Olympus, Japan), to ensure that they were free from defects.

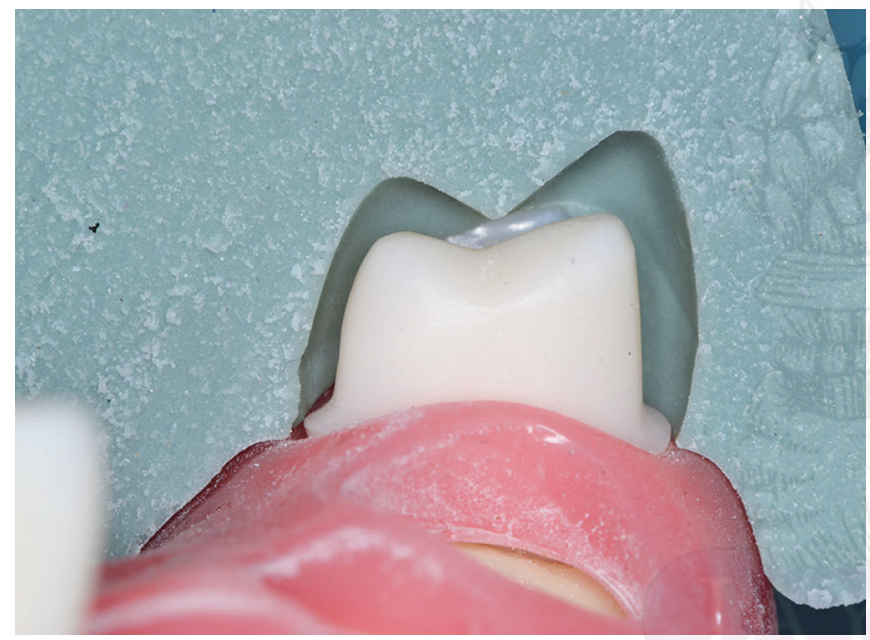

Fig. 1: The preparation with the conventional silicon guide

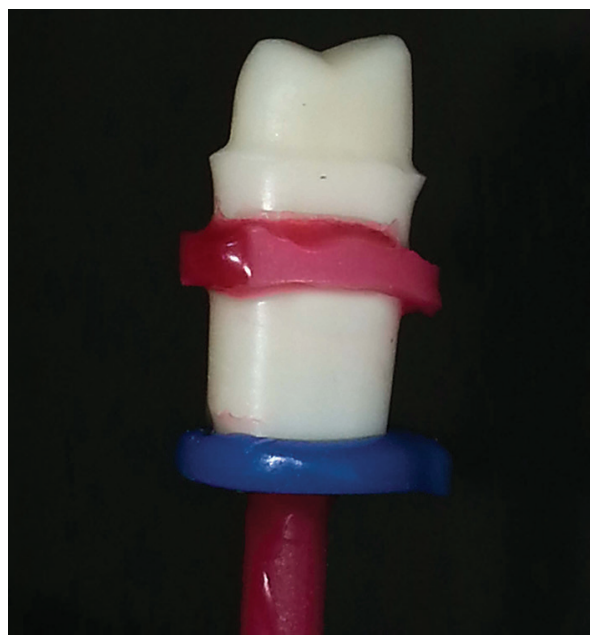

Fig. 2: The master die with the additional wax rings for better stability in the acrylic holder
Each of the prepared dies was embedded in self-cured resin (Novacryl, Tricodent LTD, Victoria Road, Burgess Hill, England) inside a brass cylinder. The dies were immersed until $3 \mathrm{~mm}$ below the margin and a custom-made positioning holder was used for stabilization during polymerization to ensure that all dies were identically positioned in their respective resin blocks (Figs 2 to 5).

\section{Preparation and Manufacturing of Crown Specimens}

The 80 specimens were divided into four groups $(n=20)$ according to the material used for the respective crowns (Table 1). Each group was further divided into two subgroups: following cementation, half of the samples were subjected to thermocycling and then cyclic loading.

Each die was digitally scanned using an intraoral scanner (OMNICAM, Cerec, Sirona). Data were exported for crown design using the in-Lab software (inLab SW4.2, Cerec, Sirona) and then sent to the milling device (inLab MCXL, Cerec, Sirona). To standardize manufacturing and avoid the need for fitting adjustments, the virtual spacing was set at $80 \mu$ on the occlusal surface and all walls until $1 \mathrm{~mm}$ above the margin and the outer design copied the shape of a similar unprepared tooth. This ensured that the 80 crowns of four materials obtained were identical in volume and thickness. For verification, the crowns were radiographed with the respective dies and the thickness was measured using the $X$-ray software (easydent 4, Vatech, Korea)

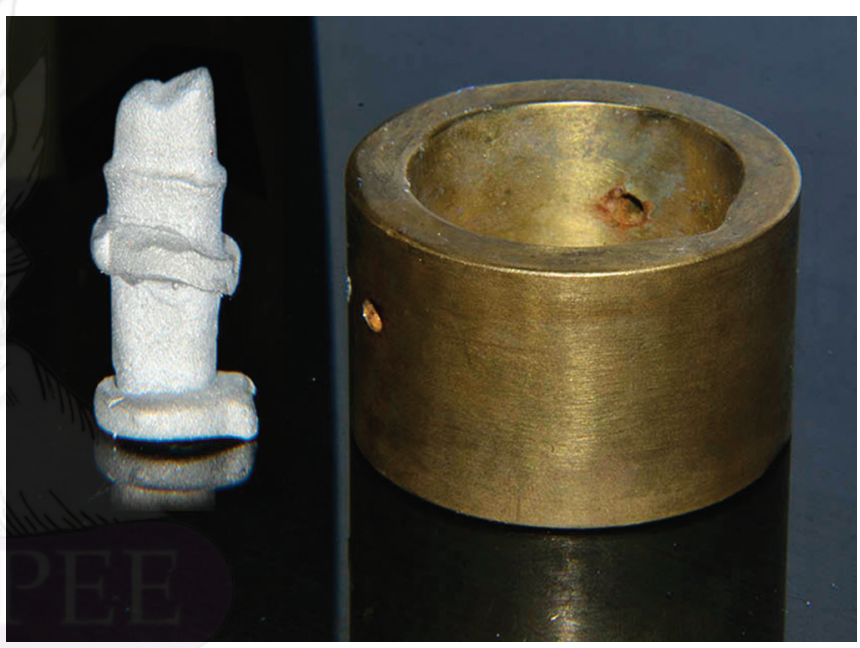

Fig. 3: Cast die and brass cylinder

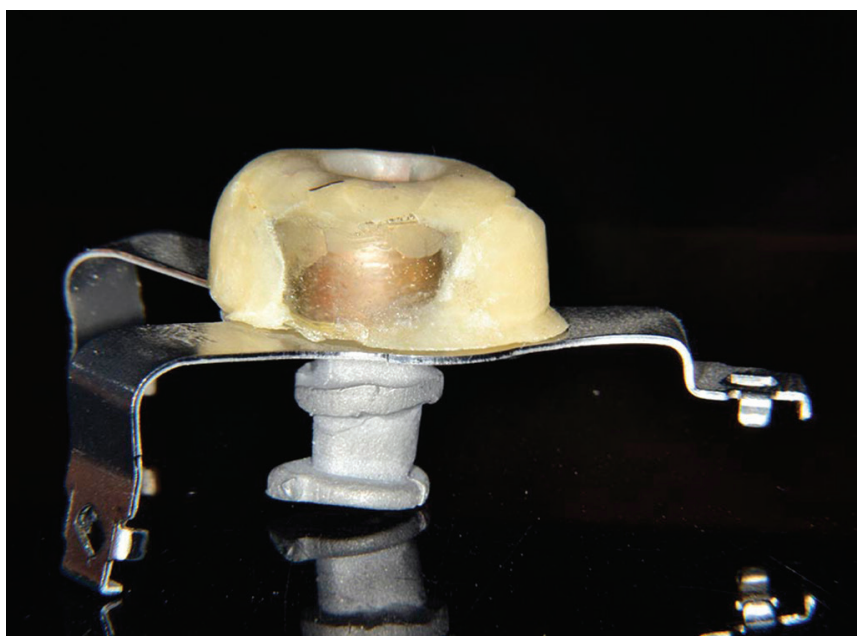

Fig. 4: Metal die(s) and positioner 


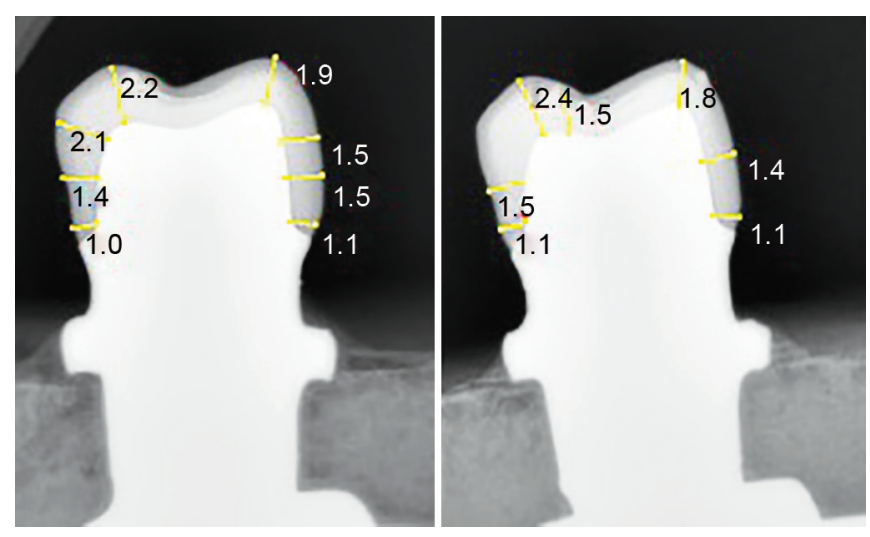

Fig. 5: The dies and respective crowns were radiographed for thickness verification

According to the manufacturer's recommendations, the crowns of groups 1, 2, and 3 were polished mechanically (Vita Enamic polishing set, VITA ZAhnfabrik, Bad Säckingen, Germany), while those of group 4 (LDGC) were crystalized and glazed in a furnace (Programat P310, Ivoclar, vivadent, Schaan, Liechtenstein).

\section{Marginal and Occlusal Gap Assessment}

The replica technique was used to evaluate the adaptation of the crowns. ${ }^{8-11}$ Each crown was filled with A-silicone lightbody impression material (express 2 light body flow, 3M ESPE, Seefeld, Germany) and placed on the corresponding die with a defined load of $20 \mathrm{~N}$ for a total period of 4 minutes using a surveyor and constant weight. Following impression material setting, the crowns were removed from the dies and the intaglios inspected for the adherence of the impression material film. Unsuccessful impressions were repeated to ensure adherence. Subsequently, a medium-bodied material with a contrasting color (express 2 regular body quick, 3M ESPE) was injected in the crown to form one piece with the film. After setting the supporting regular-bodied material, each silicone replica was removed from the crown. A sharp blade was used to segment each replica buccolingually and mesiodistally into four pieces. A blade positioner consisting of an adjusted copper band lined with pattern resin was used to standardize segmentation. The copper band had two cutting indices: one for the buccolingual cut and another for the mesiodistal cut.

Adaptation was assessed using five measurements: four cervical points (crown margin to preparation margin) and one occlusal point measured vertically at the central fossa. The thickness of the light body film at the selected points represented the respective marginal gaps in mid-buccal, mid-palatal, mid-mesial, and mid-distal and the internal gap was measured at the occlusal middle of the central groove.
All measurements were recorded using a digital optical microscope and the corresponding measuring software (portable capture pro, V2.1, Gamemax, China).

\section{Crown Cementation, Aging, and Loading}

As per the manufacturer's recommendations, only the crowns in groups 1, 2, and 3 were sandblasted using $50 \mu \mathrm{m}$ alumina powder at 1 bar pressure. All crowns (groups 1-4) were then cleaned in an ultrasonic bath containing distilled water for 4 minutes. After drying, the crowns were pretreated with a ceramic etching gel (IPS ceramic primer, Ivoclar-Vivadent) for 20 seconds, rinsed off, and air-dried. All crowns and metal dies received a universal primer (GC Multiprimer, GC, Japan).

The cementation procedure used a dual cure resin cement (GC Linkforce, GC, Japan) and was carried out under a constant pressure of $40 \mathrm{~N}$ (a surveyor with a custom-made resin tip and a constant weight was used on top). The polymerization was completed using a LEDcuring device (Elipar S10, 3M ESPE) for 20 seconds on each surface.

After cementation, half the samples within each group $(n=10)$ were subjected to thermocycling. The protocol involved 3,000 cycles of three consecutive rounds each: (1) 30 seconds at $5{ }^{\circ} \mathrm{C}$; (2) 15 seconds at ambient air temperature; and (3) 30 seconds at $55{ }^{\circ} \mathrm{C}$. The same specimens were then placed on a cyclic loading machine (CS-4 chewing simulator, Mechatronik, FeldkirchenWesterham, Germany). The tip was applied on the occlusal groove with a load of $100 \mathrm{~N}$ at a speed of $30 \mathrm{~mm} /$ minute and a frequency of $1 \mathrm{~Hz}$. No lateral sliding was programmed. A total of 200,000 cycles of load were applied to each specimen.

At the end of the fatigue procedure, the specimens were checked for possible failure or crack $^{9,12}$ using a stereoscope X20 (Olympus, Japan) with direct examination and transillumination. Failed specimens were rejected.

\section{Assessment of Fracture Load}

All specimens within the four groups were subjected to a load-tofracture test using a universal testing machine (Unitronic S205, Matest, Italy) that delivered a compressive load to the specimens at a crosshead speed of $1 \mathrm{~mm} /$ minute until failure. ${ }^{13-15}$ The $3 \mathrm{~mm}$ diameter spherical tip was located on the mesial occlusal fossa and positioned vertical to the base. Load-time curves were recorded using the testing machine's software and the load was measured in Newton. The failure load of the specimen was recorded when the force vs time graph showed an abrupt change in load, indicating a sudden decrease in the specimen's resistance to compressive loading. In correspondence to this sudden drop, load delivery underwent an automatic stop.

\section{Statistical Analysis}

One-way analysis of variance (ANOVA) tests were used to compare the gaps between the four marginal points. One-way ANOVA tests

Table 1: Materials used

\begin{tabular}{|c|c|c|c|c|c|}
\hline Group & Blocks used & Manufacturer & Composition & & Lot number \\
\hline 1 & Cerasmart & GC, Japan & Resin nanoceramic & $\begin{array}{l}\text { Composite resin material (bis-urethane } \\
\text { dimethacrylate (Bis-UDMA), dimethylaniline } \\
\text { (DMA)) } 71 \text { wt } \% \text { silica and barium glass }\end{array}$ & 1504271 \\
\hline \multirow[t]{2}{*}{2} & Enamic & VITA, Germany & PICN & Feldspar ceramic 86 wt $\%$ & 37380 \\
\hline & & & & Methacrylate polymer $14 \mathrm{wt} \%$ & \\
\hline \multirow[t]{2}{*}{3} & Brilliant Crios & COLTENE, Switzerland & Resin nanoceramic & Crosslinked methacrylates 29.3 wt $\%$ & G99755 \\
\hline & & & & Amorphous silica 70.7 wt $\%$ & \\
\hline 4 & e.max & Ivoclarvivadent Liechtenstein & LDGC & 70 vol\% lithium disilicate and glass ceramic & U50480 \\
\hline
\end{tabular}


followed by post hoc tests were employed to compare the margin and occlusal gaps between groups. Univariate analyses of variance were used to study the effect of material and aging on the the fracture load value. Pearson correlations were performed to assess the relationship between gap magnitude and resistance to fracture.

The IBM $^{\circledast}$ SPSS $^{\circledast}$ statistics 23.0 statistical package was used to carry out all statistical analyses. Statistical significance was set at 0.05 .

\section{Results}

\section{Gap Measurements}

The mean marginal gaps ranged between $68.5 \pm 23.8 \mu \mathrm{m}$ and $87 \pm 29.1 \mu \mathrm{m}$. Within each of the four groups, a one-way ANOVA test showed that there were no statistically significant differences between the four assessed marginal locations $(0.13<a<0.91$; Table 2$)$.

The marginal gaps were the highest in group 1, followed by group 4 and then finally by groups 2 and 3 ; there was a statistically significant difference only between groups 1 and 3 ( $\alpha=0.048$; Table 3 ).

Occlusal gaps ranged from $220.7 \pm 33.3 \mu \mathrm{m}$ to $275.5 \pm 46.5 \mu \mathrm{m}$; the largest gap being associated with group 1, followed by group 2 , then group 3 , and finally group 4 (Table 3 ). The only significant difference was between groups 1 and $4(a=0.01)$.

\section{Fracture Load}

Two specimens were rejected due to fracture following cyclic loading (one from group 1 and another from group 2).

Fracture loads ranged from $633.8 \pm 127.3 \mathrm{~N}$ to $1596.4 \pm 497.7 \mathrm{~N}$. The highest values were recorded for group 4, followed by group 2 , then group 3, and finally by group 1 (Graph 1). The pairwise comparison of the fracture resistance between groups showed that the fracture load recorded for group 4 was significantly higher than those for group $1(a<0.001)$, group $2(a=0.017)$, and group 3 $(a<0.001)$. Fracture resistance was significantly higher for group 2 compared to that for group $1(a=0.01)$, whereas group 3 displayed similar results to groups 1 and $2(a>0.05$; Table 4$)$.

\section{Correlations and Associated Factors}

Univariate analysis of the effects of material and aging showed that material had a significant effect on the fracture load ( $a=0.001)$, while aging had no significant effect $(\alpha=0.41)$ and the combined effect of material and aging was not statistically significant $(a=0.29$; Table 5).

Pearson correlations between the gap magnitude and fracture load ranged from weak for the occlusal gaps (Pearson $R=-0.3$; $p=0.007$ ) to nonexistent in the marginal mid-buccal gaps (Pearson $R=-0.004 ; p=0.972 ;$ Table 6).

\section{Discussion}

This study involves the fabrication of identical crowns on identical metal dies, this was to investigate one variance between groups
Table 3: Multiple comparison of gaps between groups

\begin{tabular}{llclll}
\hline & & \multicolumn{3}{l}{ Marginal gap } & \multicolumn{3}{l}{ Occlusal gap } \\
\cline { 3 - 6 } Material & & $\begin{array}{l}\text { Mean } \\
\text { difference }\end{array}$ & p value & difference & p value \\
\hline Cerasmart & Enamic & 8.06 & 0.359 & 24.05 & 0.457 \\
& Brilliant & $11.49^{*}$ & $\mathbf{0 . 0 4 8}^{*}$ & 33.40 & 0.088 \\
& E.max & 3.23 & 1.00 & $54.85^{*}$ & $\mathbf{0 . 0 0 1 *}$ \\
Enamic & Brilliant & 3.43 & 1.00 & 9.35 & 1.00 \\
& E.max & -4.84 & 1.00 & 30.80 & 0.144 \\
Brilliant & E.max & -8.26 & 0.323 & 21.45 & 0.678 \\
\hline
\end{tabular}

* indicates where the difference was significant

Table 4: Pairwise comparisons of fracture loads between groups

\begin{tabular}{llll}
\hline Material & Material & Mean difference & $p$ value \\
\hline Cerasmart & Enamic & -449.76 & $\mathbf{0 . 0 1 *}$ \\
& Brilliant & -248.33 & 0.156 \\
\multirow{2}{*}{ Enamic } & E.max & -787.58 & $\mathbf{0 . 0 0 0 ^ { * }}$ \\
& Brilliant & 201.42 & 0.416 \\
Brilliant & E.max & -337.83 & $\mathbf{0 . 0 1 7 ^ { * }}$ \\
\hline
\end{tabular}

* indicates where the difference was significant

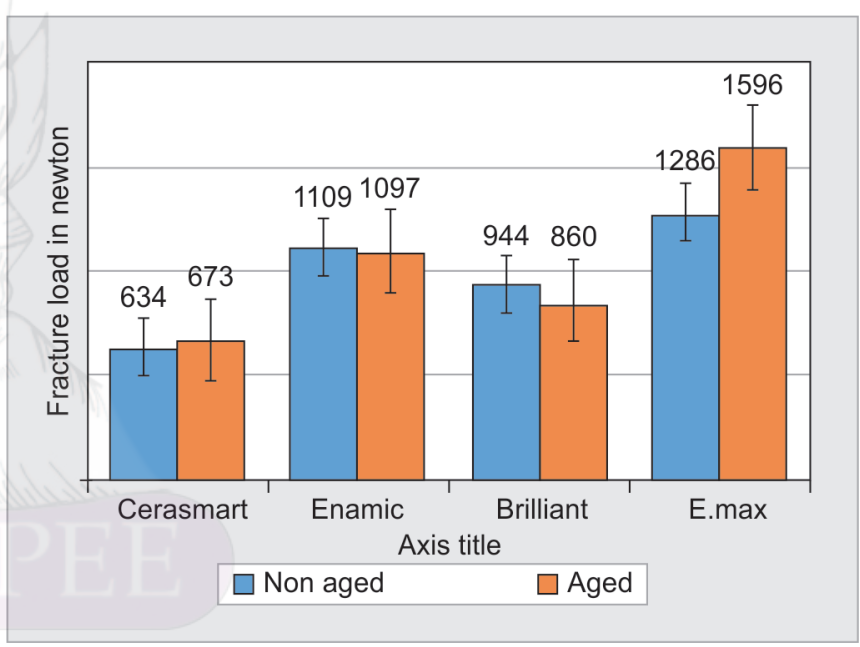

Graph 1: Fracture load of groups with and without aging

Table 5: Tests of between-subjects effects on fracture load

\begin{tabular}{lrl}
\hline Source & \multicolumn{1}{l}{ F } & Sig. \\
\hline Aging & 0.68 & 0.41 \\
Material & 18.67 & $\mathbf{0 . 0 0 1 *}$ \\
Aging $\times$ material & 1.28 & 0.29 \\
\hline
\end{tabular}

* indicates where the difference was significant

Table 2: Mean and comparison of gaps

\begin{tabular}{|c|c|c|c|c|c|c|c|}
\hline \multirow[b]{3}{*}{ Groups } & \multicolumn{5}{|l|}{ Region } & \multicolumn{2}{|c|}{ Within groups } \\
\hline & \multirow{2}{*}{$\begin{array}{l}\text { Occlusal }(n=20) \\
\text { Mean }\end{array}$} & \multirow{2}{*}{$\begin{array}{l}\text { Buccal }(n=20) \\
\text { Mean }\end{array}$} & \multirow{2}{*}{$\begin{array}{l}\text { Palatal }(n=20) \\
\text { Mean }\end{array}$} & \multirow{2}{*}{$\begin{array}{l}\text { Mesial }(n=20) \\
\text { Mean }\end{array}$} & \multirow{2}{*}{$\begin{array}{l}\text { Distal }(n=20) \\
\text { Mean }\end{array}$} & \multicolumn{2}{|c|}{ Margin comparison ANOVA } \\
\hline & & & & & & $F$ & $p$ value \\
\hline Cerasmart & $275.50 \pm 46.5$ & $82.9 \pm 31.1$ & $79.4 \pm 33.1$ & $87 \pm 29.1$ & $80 \pm 32.3$ & 0.23 & 0.87 \\
\hline Enamic & $251.45 \pm 46.1$ & $74.8 \pm 24.3$ & $68.5 \pm 23.8$ & $76.5 \pm 28.2$ & $77.3 \pm 27.7$ & 0.48 & 0.7 \\
\hline Brilliant & $242.10 \pm 42.0$ & $73.1 \pm 23.3$ & $68.8 \pm 20.7$ & $70.8 \pm 2$ & $70.7 \pm 16.8$ & 0.18 & 0.91 \\
\hline E.MAX & $220.65 \pm 33.3$ & $84 \pm 18.2$ & $82.7 \pm 21.1$ & $75.4 \pm 18.4$ & $74.4 \pm 15.7$ & 1.99 & 0.13 \\
\hline
\end{tabular}


Table 6: Load to gaps correlations $n=78$

\begin{tabular}{lccccc}
\hline Fract_load & Occlusal & Buccal & Palatal & Mesial & Distal \\
\hline $\begin{array}{l}\text { Pearson } \\
\text { correlation }\end{array}$ & $-0.305^{* *}$ & -0.004 & -0.084 & $-0.241^{*}$ & -0.135 \\
$p$ value & 0.007 & 0.972 & 0.462 & 0.034 & 0.239 \\
\hline
\end{tabular}

* and ${ }^{* *}$ shows no correlation as $R<-0.5$

which is the materials used, and the details investigated were the internal fit and the fracture load upon occlusal pressure. Half of the groups were artificially aged to investigate another variance (the aging) within each group. Finally, this study searched for a relation between the gap magnitude and the fracture load.

Although many authors use a $6 \mathrm{~mm}$ diameter tip for the fracture test, a smaller diameter was used in this study $(3 \mathrm{~mm})$ to mimic the approximate size of an antagonist cusp, ${ }^{16-18}$ the virtual die spacer was set at $80 \mu^{19}$ because the preparation was done using a surveyor and the $6^{\circ}$ taper of the preparation would cause excessive stress on the crowns if less relief was chosen. ${ }^{19,20}$

The use of metal dies rather than natural teeth in the present study ensured the presence of adequate and standardized support for testing the selected properties of the restorative materials. Natural teeth differ in morphology, quality, age, and storage time because, practically, they cannot all be extracted on the same day. ${ }^{18}$ With the increased speed with which new materials are released into the market, in vitro studies remain the only short-term alternative to provide rapid feedback that approximates the clinical reality in order to assist clinicians in their choices of restorative material for their patients.

The marginal gaps recorded in the four CAD/CAM ceramic materials assessed in this study were all within the range of clinical acceptance $(<120 \mu \mathrm{m})^{21,22}$ and the values reached for fracture in all groups were above the ranges recorded for occlusal forces in the posterior region $(>500 \mathrm{~N}$ ). In light of the data, we failed to reject the null hypothesis concerning the internal fit of the four groups but rejected the null hypothesis with regard to fracture resistance since significantly greater values were recorded for e.max. Finally, we failed to reject the final null hypothesis of no association between internal fit and fracture resistance.

Although nonsignificant, the same work flow resulted in small differences in marginal adaptation between the four ceramics; occlusal gap magnitude following the sequence e.max $<$ Brilliant $<$ Enamic $<$ Cerasmart and marginal gap magnitude in the order of Brilliant < Enamic < e.max < Cerasmart. Except for Cerasmart, our data support the results reported by Goujat et al. ${ }^{6}$ where e.max and Cerasmart had gap magnitudes that were equal to each other and smaller than that of Enamic. The fact that the different RNC materials exhibited differences in gap values may be the result of variations in the resin matrix composition, size, and type of the particles used as charge, ${ }^{6}$ these dispersed particles on the milled surface are easily washed away with the sandblasting and hydrofluoric acid treatment. Bottino, ${ }^{8}$ on the contrary, found higher gap magnitudes for feldspathic ceramic restorations than PICN, probably due to the weaker charge in feldspathic ceramic and coarse surface on such a structure; several previous studies ${ }^{5,23,24}$ showed that milling resin ceramics yields a smoother surface and better margin integrity than glass ceramics and, although this might have clinical relevance in thin structures, such as veneers, it did not show a significant difference for full contoured restorations in the present study.

Data from previous studies suggest that the pulpal surface exhibits greater gap magnitudes compared to marginal gaps, $1,6,21,25$ a conclusion that is supported by the results of the present study even though the differences recorded did not reach statistical significance in all four assessed groups.

The larger fracture resistance recorded for e.Max is likely due to the interpenetrating needle-type lithium disilicate that responds better to load than the dispersed spherical charge in RNC or the fused particles of PICN; this result is in agreement with previously published results, ${ }^{6,18,26-30}$ and the literature highlights that lithium disilicate has clearly higher fracture strength than other ceramic groups. ${ }^{6,18,26,28-30}$ PICN with fused particles proved having higher strength than RNC with dispersed fillers. According to Stawarczyk, the fact that PICN has higher resistance than RNC but lower than LDGC comes with the same order in hardness and wear properties to the antagonist teeth. ${ }^{31}$ The higher charge percentage with an interpenetrating network puts PICN in the midrange between LDGC and RNC with dispersed fillers, this was also confirmed by Albero. ${ }^{32}$

The results in the present study of 3,000 cycles thermocycling equivalent to 2.5 years of clinical use $\mathrm{s}^{33}$ did not significantly affect fracture resistance of any of the groups, this comes in contrast to the works of Sonmez et al. ${ }^{29}$ and Aboushelib et al. ${ }^{34}$ who found that PICN and RNC are more sensitive to thermocycling than LDGC. Supporting previous reports, gap magnitude showed no significant correlations with fracture load, ${ }^{6,20}$ thereby suggesting that the resistance to fracture is mainly related to the material factor.

Various other factors may interfere with the results, such as the setting of the design software ${ }^{35}$ for the virtual die spacer and the gap evaluation technique used. A recent study ${ }^{25}$ showed that optical coherence tomography (OCT) can give lower values than the replica technique for the same prosthetic work. The replica technique may be sensitive to the taper angle, the height of the preparation, the viscosity of the light body used, and the pressure applied. A comparison with OCT and microtomography (micro-CT) might be useful in further studies.

\section{CONCLUSION}

Within the limits of this study, the following conclusions can be drawn:

- PICN and RNC materials appear to equally withstand the occlusal forces in normal posterior occlusion.

- PICN and RNC may be used for single unit restorations with promising prognosis.

- Thermodynamic aging does not seem to weaken PICN and RNC materials.

\section{References}

1. Pradies G, Zarauz C, et al. Clinical evaluation comparing the fit of all-ceramic crowns obtained from silicone and digital intraoral impressions based on wavefront sampling technology. J Dent 2015;43(2):201-208. DOI: 10.1016/j.jdent.2014.12.007.

2. Vichi $A$, Louca $C$, et al. Color related to ceramic and zirconia restorations: a review. Dent Mater 2011;27(1):97-108. DOI: 10.1016/ j.dental.2010.10.018.

3. van den Breemer CR, Vinkenborg C, et al. The Clinical Performance of Monolithic Lithium Disilicate Posterior Restorations After 5, 10, and 15 Years: A Retrospective Case Series. Int J Prosthodont 2017;30(1):62-65. DOI: 10.11607/ijp.4997.

4. Mainjot AK, Dupont NM, et al. From Artisanal to CAD-CAM Blocks: State of the Art of Indirect Composites. J Dent Res 2016;95(5):487-495. DOI: $10.1177 / 0022034516634286$.

5. Argyrou R, Thompson GA, et al. Edge chipping resistance and flexural strength of polymer infiltrated ceramic network and resin 
nanoceramic restorative materials. J Prosth Dent 2016;116(3):397-403. DOI: 10.1016/j.prosdent.2016.02.014.

6. Goujat $A$, Abouelleil $H$, et al. Mechanical properties and internal fit of 4 CAD-CAM block materials. J Prosth Dent 2018;119(3):384-389. DOI: 10.1016/j.prosdent.2017.03.001.

7. El Zhawi H, Kaizer MR, et al. Polymer infiltrated ceramic network structures for resistance to fatigue fracture and wear. Dent Mater 2016;32(11):1352-1361. DOI: 10.1016/j.dental.2016.08.216.

8. Bottino MA, Campos F, et al. Inlays made from a hybrid material: adaptation and bond strengths. Oper Dent 2015;40(3):E83-E91. DOI: 10.2341/13-343-L.

9. Zhao K, Wei YR, et al. Influence of veneer and cyclic loading on failure behavior of lithium disilicate glass-ceramic molar crowns. Dent Mater 2014;30(2):164-171. DOI: 10.1016/j.dental.2013.11.001.

10. RahmeHY, Tehini GE, et al. In vitro evaluation of the "replica technique" in the measurement of the fit of Procera crowns. J Contemp Dent Pract 2008;9(2):25-32.

11. Laurent M, Scheer $P$, et al. Clinical evaluation of the marginal fit of cast crowns - validation of the silicone replica method. J Oral Rehabil 2008;35(2):116-122. DOI: 10.1111/j.1365-2842.2003.01203.x.

12. Belli R, Geinzer $E$, et al. Mechanical fatigue degradation of ceramics versus resin composites for dental restorations. Dent Mater 2014;30(4):424-432. DOI: 10.1016/j.dental.2014.01.003.

13. Aboushelib MN, de Jager $N$, et al. Effect of loading method on the fracture mechanics of two layered all-ceramic restorative systems. Dent Mater 2007;23(8):952-959. DOI: 10.1016/j. dental.2006.06.036.

14. Donnelly TJ, Burke FJ. In vitro failure of crowns produced by two CAD/ CAM systems. Eur J Prosthodont Restor Dent 2011;19(3):111-116.

15. Wang XD, Jian $Y T$, et al. Effect of core ceramic grinding on fracture behaviour of bilayered lithium disilicate glass-ceramic under two loading schemes. J Dent 2014;42(11):1436-1445. DOI: 10.1016/ j.jdent.2014.03.014

16. Johansson C, Kmet $G$, et al. Fracture strength of monolithic allceramic crowns made of high translucent yttrium oxide-stabilized zirconium dioxide compared to porcelain-veneered crowns and lithium disilicate crowns. Acta Odontol Scand 2014;72(2):145-153. DOI: 10.3109/00016357.2013.822098.

17. Dietschi $D$, Maeder $M$, et al. In vitro resistance to fracture of porcelain inlays bonded to tooth. Quintessence Int 1990;21(10): 823-831.

18. Sagsoz O, Yildiz M, et al. In vitro Fracture strength and hardness of different computer-aided design/computer-aided manufacturing inlays. Niger J Clin Pract 2018;21(3):380-387.

19. Rekow $D$, Thompson VP. Engineering long term clinical success of advanced ceramic prostheses. J Mater Sci Mater Med 2007;18(1): 47-56. DOI: 10.1007/s10856-006-0661-1.

20. Liu B, Lu C, et al. The effects of adhesive type and thickness on stress distribution in molars restored with all-ceramic crowns. J Prosthodont 2011;20(1):35-44. DOI: 10.1111/j.1532-849X.2010.00650.x.
21. Carbajal Mejia JB, Yatani $\mathrm{H}$, et al. Marginal and Internal Fit of CAD/CAM Crowns Fabricated Over Reverse Tapered Preparations. J Prosthodont 2017; (Epub ahead of print). DOI: 10.1111/jopr.12715.

22. Scherrer SS, de Rijk WG, et al. Effect of cement film thickness on the fracture resistance of a machinable glass-ceramic. Dent Mater 1994;10(3):172-177.

23. Awada A, Nathanson D. Mechanical properties of resin-ceramic CAD/ CAM restorative materials. J Prosthet Dent 2015;114(4):587-593. DOI: 10.1016/j.prosdent.2015.04.016.

24. Tsitrou EA, Northeast $S E$, et al. Brittleness index of machinable dental materials and its relation to the marginal chipping factor. J Dent 2007;35(12):897-902. DOI: 10.1016/j.jdent.2007.07.002.

25. Ayse Gozde T, Metin S, et al. Evaluation of adaptation of ceramic inlays using optical coherence tomography and replica technique. Braz Oral Res 2018;32:e005. DOI: 10.1590/1807-3107BOR-2018.vol32.0005.

26. Al-Akhali M, Chaar MS, et al. Fracture resistance of ceramic and polymer-based occlusal veneer restorations. J Mech Behav Biomed Mater 2017;74:245-250. DOI: 10.1016/j.jmbbm.2017.06.013.

27. Eldafrawy M, Ebroin MG, et al. Bonding to CAD-CAM Composites: An Interfacial Fracture Toughness Approach. J Dent Res 2018;97(1):60-67. DOI: 10.1177/0022034517728714.

28. Sagsoz NP, Yanikoglu N. Evaluation of the fracture resistance of computer-aided design/computer-aided manufacturing monolithic crowns prepared in different cement thicknesses. Niger J Clin Pract 2018;21(4):417-422. DOI: 10.4103/njcp.njcp_137_17.

29. Sonmez N, Gultekin P, et al. Evaluation of five CAD/CAM materials by microstructural characterization and mechanical tests: a comparative in vitro study. BMC Oral Health 2018;18(1):5. DOI: 10.1186/s12903-0170458-2.

30. Wendler M, Belli R, et al. Chairside CAD/CAM materials. Part 3: Cyclic fatigue parameters and lifetime predictions. Dent Mater 2018;34(6): 910-921. DOI: 10.1016/j.dental.2018.03.024.

31. Stawarczyk B, Liebermann A, et al. Evaluation of mechanical and optical behavior of current esthetic dental restorative CAD/CAM composites. J Mech Behav Biomed Mater 2015;55:1-11. DOI: 10.1016/ j.jmbbm.2015.10.004.

32. Albero A, Pascual A, et al. Comparative characterization of a novel CAD-CAM polymer-infiltrated-ceramic-network. J Clin Exp Dent 2015;7(4):e495-e500. DOI: 10.4317/jced.52521.

33. Ehlers V, Kampf G, et al. Effect of thermocycling with or without 1 year of water storage on retentive strengths of luting cements for zirconia crowns. J Prosthet Dent 2015;113(6):609-615. DOI: 10.1016/ j.prosdent.2014.12.001.

34. Aboushelib MN, Elsafi MH. Survival of resin infiltrated ceramics under influence of fatigue. Dent Mater 2016;32(4):529-534. DOI: 10.1016/ j.dental.2015.12.001.

35. Shim JS, Lee JS, et al. Effect of software version and parameter settings on the marginal and internal adaptation of crowns fabricated with the CAD/CAM system. J Appl Oral Sci 2015;23(5):515-522. DOI: 10.1590/1678-775720150081. 